\title{
Erwartungen und Kenntnisse von Ausbildungskandidaten in Verhaltenstherapie zu Psychotherapienebenwirkungen
}

\author{
Marie-Luise Schermuly-Haupt ${ }^{a} \quad$ Michael Linden $^{b, c}$ \\ ${ }^{a}$ Abteilung für psychische und psychosomatische Erkrankungen am Reha-Zentrum Seehof der Deutschen \\ Rentenversicherung Bund, Teltow, Deutschland; ${ }^{\mathrm{b}}$ Charité Universitätsmedizin Berlin, Klinik für Innere Medizin \\ mit Schwerpunkt Psychosomatik, Forschungsgruppe Psychosomatische Rehabilitation, Berlin, Deutschland; \\ 'Institut für Verhaltenstherapie Berlin, Berlin, Deutschland
}

\section{Schlüsselwörter \\ Psychotherapieausbildung · Nebenwirkungen · Qualitätssicherung}

\begin{abstract}
Zusammenfassung
Hintergrund: Die Aufklärung, Erkennung, Vorbeugung und Bearbeitung von Psychotherapienebenwirkungen stellt hohe Anforderungen an Therapeuten. Grundsätzlich gilt zudem, dass es psychologische Barrieren gibt, negative therapeutische Entwicklungen wahrzunehmen und dem eigenen therapeutischen Handeln zuzuordnen. Dazu trägt bei, dass dieses Thema bislang in der Ausbildung eher wenig Beachtung findet und kaum systematisch abgehandelt wird. Um den eventuellen Handlungsbedarf abschätzen zu können, sind Daten von Interesse zu den einschlägigen Einstellungen und Kenntnissen von Therapeuten in Ausbildung. Methode: In einem halbstrukturierten Interview wurden 100 Psychotherapeuten in Ausbildung mit dem Schwerpunkt Verhaltenstherapie zu Nebenwirkungen ihrer Arbeit befragt. Die Therapeuten machten Angaben zur Qualität, Häufigkeit und den möglichen Ursachen von Psychotherapienebenwirkungen. Ergebnisse: Therapeuten in Ausbildung rechnen in etwa jedem 2. Fall mit dem Auftreten von Nebenwirkungen. Sie erwarten vor allem Symptomverschlechterung und negative Entwicklungen im sozialen Netz. Es zeigt sich eine Tendenz, das eigene Verfahren und die eigenen Behandlungen für weniger riskant zu halten als die der Kollegen. Ursachen von Nebenwirkungen sehen die Therapeuten vor allem in der therapeutischen Beziehung sowie The-
\end{abstract}

rapeutenvariablen und weniger in Patientencharakteristika. Schlussfolgerung: Psychotherapeuten in Ausbildung sind sich dem Problem der Nebenwirkungen durchaus bewusst, was ein guter Ansatzpunkt für die Vermittlung entsprechender Ausbildungsinhalte ist. An den Aus- und Weiterbildungsinstituten sollten Seminare zum Problem der Psychotherapienebenwirkungen zum Standard gehören.

c 2020 S. Karger AG, Basel

\section{Expectations and Knowledge of Behavioral Therapy} Trainees in Regard to Psychotherapy Side Effects

\section{Keywords}

Psychotherapy training · Side effects · Quality assurance

\section{Abstract}

Background: The recognition, prevention, and management of psychotherapy side effects are challenging for mental health professionals. There are psychological barriers to recognize negative therapeutic developments and accept a relation to one's own therapeutic actions. This topic has so far received little attention in the training of psychotherapists. The goal of this study is to assess attitudes and knowledge of psychotherapists in training, a prerequisite for educational measures. Methods: In a semi-structured interview, 100 cognitive behavioral therapists in training were asked about their knowledge and experiences with side effects of psychotherapy, the ex- 
pected frequencies, examples, and possible causal fac tors. Results: Deterioration of symptoms and changes in the social net are the most frequent side effects, as expected by the therapists. They expect side effects in about every second case. They perceive their own therapy and those with the same theoretical orientation (cognitive behavioral therapy) as less risky than those of other therapists and therapy schools. Therapists see the therapeutic relationship as the most important causal factor for psychotherapy side effects and think that therapist variables are more significant than patient variables. Conclusions: Psychotherapists in training are well aware of the problem of side effects. This is a good starting point for teaching about this subject. Seminars on psychotherapy side effects should be standard in the education of psychotherapists.

○ 2020 S. Karger AG, Base

\section{Hintergrund}

Es gehört zu den grundsätzlichen Pflichten von Therapeuten, das Selbstbestimmungsrecht ihrer Patienten zu wahren. Daher müssen sie auch über die Risiken der geplanten Therapie informieren. Dies ist u.a. durch das $\mathrm{Pa}-$ tientenrechtegesetz, Berufsrecht, Haftungsrecht und sogar Strafrecht festgelegt [BPTK, 2013]. Grundsätzlich ist davon auszugehen, dass alles, was wirkt, auch Nebenwirkungen haben kann und dass dies verfahrensinhärente Probleme sind. Je nach Art der Erhebungsmethodik und Problemdefinition werden Inzidenzraten zwischen 5 und 94\% berichtet, wobei schon 5\% im Vergleich beispielsweise zur Psychopharmakotherapie eine durchaus relevante Größenordnung ist [Dishion et al., 1999; Mayou et al., 2000; Roback, 2000; Strauß und Eckert, 2001; Lambert und Ogles, 2004; Moos, 2005; Lilienfeld, 2007; Berk und Parker, 2009; Barlow, 2010; Pence et al., 2010; Warren et al., 2010; Jacobi et al., 2011; Lambert, 2011; Brakemeier et al., 2012; Nelson und Hiller, 2013; Linden et al., 2015; Hermanutz, 2017; Schermuly-Haupt et al., 2018]. Darauf zu achten ist ein wichtiger Teil der Qualitätssicherung in der Psychotherapie.

Da Psychotherapie eine von Therapeuten persönlich erbrachte Leistung ist, gibt es im Umgang mit dem Nebenwirkungsthema auch therapeutenseitig einige Probleme. Es ist mit einem Wahrnehmungsbias zu rechnen in Form einer Tendenz, das Nebenwirkungsthema eher auszublenden, Verschlechterungen der Patienten nicht wahrzunehmen oder external zu attribuieren [Dunning et al., 2004; Hannan et al., 2005; Boisvert und Faust, 2006; Hatfield et al., 2010; Lambert, 2010; Dobson und Beshai, 2013; Schermuly et al., 2014] und die Ergebnisse der eigenen Arbeit eher positiv zu konnotieren [Lambert, 2010; Walfish et al., 2012]. Aus der Prävalenz von Psychothera- pienebenwirkungen, ihrer Relevanz für den Patienten und den Problemen bei der Erfassung ergibt sich die Notwendigkeit, dem Thema der Nebenwirkungen in der Ausbildung von Psychotherapeuten angemessene Aufmerksamkeit zu widmen. In einer Befragung schwedischer Psychotherapeuten berichteten nur 11\%, mit dem Thema Psychotherapienebenwirkungen bereits in ihrer Ausbildung konfrontiert worden zu sein oder aus der Literatur davon erfahren zu haben [Bystedt et al., 2014]. Es scheint auch an methodischem Wissen zu fehlen, wie solche negativen Effekte erfasst werden könnten. Das Thema wird auch im Ausbildungskatalog für psychologische Psychotherapeuten (PsychTh-APrV) nicht erwähnt. Wenn das Thema in der Ausbildung aber nicht vorkommt, dann werden Psychotherapeuten diesbezüglich unvorbereitet in die Berufspraxis entlassen, was einige der vorgenannten Probleme bezüglich des Nebenwirkungsmanagements erklären kann. Psychotherapeuten in Ausbildung erzielen ohne Zweifel nachweisbare positive Effekte für ihre Patienten [Hasenauer und Tschuschke, 2017], es sollte aber schon früh auf eine Sensibilisierung für mögliche Nebenwirkungen des eigenen Handelns geachtet werden. Solide diesbezügliche Kenntnisse ermöglichen gegebenenfalls Nebenwirkungen vorzubeugen oder auch ein fachgerechtes Nebenwirkungsmanagement.

Auf diesem Hintergrund ist von Interesse, welche Voreinstellungen und Kenntnisse Psychotherapeuten in Ausbildung mitbringen, als Grundlage für eine Planung von Ausbildungskursen zu diesem Thema. In der vorliegenden Studie sollte geklärt werden, welche Arten von Nebenwirkungen Psychotherapeuten in Ausbildung kennen oder erwarten, wie hoch sie die Auftretenswahrscheinlichkeit einschätzen, welche Ursachen von Nebenwirkungen ihnen bekannt sind und wie risikobehaftet sie das Psychotherapieverfahren, das sie gerade lernen, im Vergleich zu anderen Verfahren einschätzen.

\section{Methode}

\section{Teilnehmer}

In drei Ausbildungsinstituten mit dem Schwerpunkt Verhaltenstherapie in Berlin und Brandenburg wurde uns ermöglicht, eine Befragung von Psychologischen Psychotherapeuten in Ausbildung durchzuführen. Am Institut für Verhaltenstherapie Berlin (IVB) haben 68, am Zentrum für Psychotherapie der Humboldt Universität zu Berlin (ZPHU) 22 und am Institut für Verhaltenstherapie Brandenburg (IVT) 10 Ausbildungskandidaten teilgenommen. Insgesamt haben sich 96 Diplompsychologen und vier approbierte Mediziner in Ausbildung zum Verhaltenstherapeuten an der Untersuchung beteiligt. Alle Ausbildungskandidaten haben bereits unter Supervision Patienten behandelt. Die Rekrutierung der Teilnehmer erfolgte über Aushänge, E-Mail-Verteiler und kurze Vorstellungen des Projektes in Seminaren. Die Therapeuten wurden über die Ziele und den Ablauf der Untersuchung aufgeklärt und gaben eine schriftliche Einwilligung. 
Unzureichendes Ergebnis/ Misserfolg

Verlängerung der Behandlung

Komplianzprobleme

Neue Symptome

Verschlechterung von Symptomen

Belastung/ Unwohlsein

Spannungen in der Therapiebeziehung

Abhängigkeit vom Therapeuten

Familiäre Probleme

Familiäre Veränderungen

Veränderungen des sozialen Netzes

Berufliche Probleme

Berufliche Veränderungen

Arbeitsunfähigkeit

Änderungen der Lebenssituation

Missbrauch der Therapie

Stigmatisierung

sonstiges

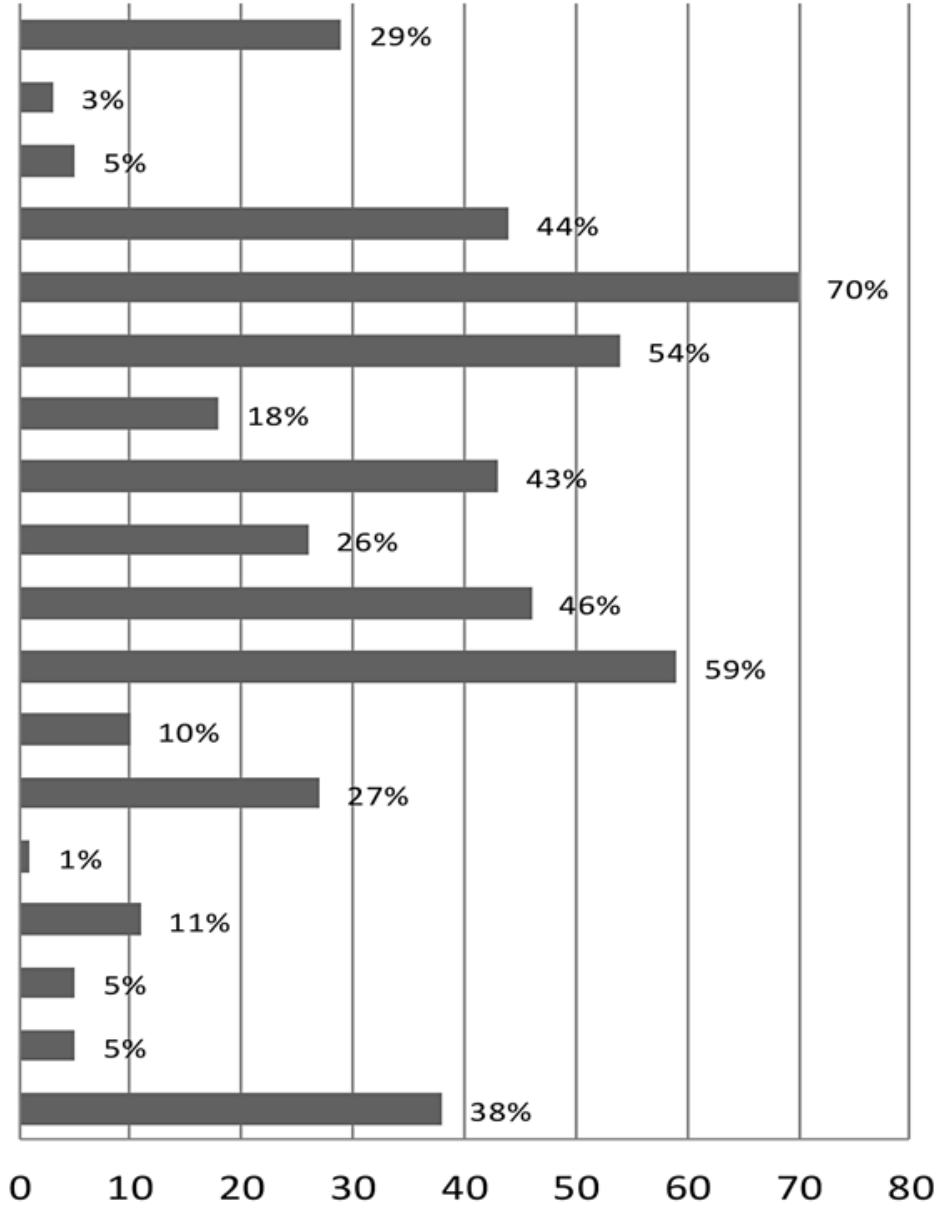

Abb. 1. Spontannennungen von Psychotherapienebenwirkungen durch Therapeuten $(N=100)$ zugeordnet zu den Kategorien der UE-ATR Checkliste.

Instrumente und Untersuchungsdurchführung

Die Therapeuten wurden zunächst um Angaben zu soziodemografischen Variablen (Alter, Geschlecht etc.) und zum Ausbildungshintergrund (z.B. Ausbildungsjahr, Berufserfahrung, Anzahl abgeschlossener Fälle etc.) gebeten. Es wurde ihnen dann eine Definition vorgelesen, welche Psychotherapienebenwirkungen als unerwünschte, aber auch unvermeidliche Begleiterscheinung einer korrekt durchgeführten Psychotherapie beschreibt [Linden und Strauss, 2018; Linden et al., 2018].

Sie wurden dann um 6 Beispiele (freie Nennungen) für mögliche Nebenwirkungen gebeten. Diese wurden von zwei unabhängigen Ratern kategorisiert in Anlehnung an die UE-ATR Checkliste [Linden und Haupt, 2012; Linden, 2013]. Es war möglich, 92\% der Beispielnennungen zuzuordnen. Cohens Kappa für die Beurteilerübereinstimmung zwischen den zwei Ratern lag bei $\kappa=0,75$ ( $p<0,001$ ), was für eine gute Übereinstimmung bei der Zuordnung spricht [Wirtz und Caspar, 2002].

Im nächsten Schritt wurden folgende Fragen gestellt, die von den Therapeuten mit Prozentangaben zu beantworten waren:

"In wie vielen Fällen treten Nebenwirkungen von Psychotherapie generell auf?"

"In wie vielen Fällen treten Nebenwirkungen von Verhaltenstherapie auf?"
"In wie vielen Fällen traten Nebenwirkungen bei eigenen Fällen auf?"

"Treten in der Verhaltenstherapie eher mehr, gleich viel oder weniger Nebenwirkungen auf als in der Psychoanalyse/Gesprächspsychotherapie?"

Die Therapeuten wurden darüber hinaus gebeten anzugeben, was sie als vorrangige Risikofaktoren für die Entwicklung von $\mathrm{Ne}$ benwirkungen ansehen. Dazu wurde ihnen in Anlehnung an Frohburg [2002] eine Liste vorgelegt mit den Items: Schwere und Dauer der Störung, kritische Lebensereignisse, Diagnose/Indikation, Störungsbild, Fallkonzeption, Therapiemethoden und technische Fehler, fachlich-theoretische Qualifikation, praktische Erfahrung und eigene Konflikte des Therapeuten, Persönlichkeit des Patienten und des Therapeuten, Therapeut-Patient-Beziehung, unzureichende Supervision, gesetzliche Rahmenbedingungen und externe Faktoren. Die Antworten mit den Beurteilungsstufen "auf keinen Fall", "eher nicht", "eher ja” oder "auf jeden Fall” erlauben eine Einschätzung dazu, ob die Therapeuten in Ausbildung das Auftreten von Nebenwirkungen eher dem eigenen Verhalten oder der Krankheit oder sonstigen Einflüssen zuschreiben. 
Tabelle 1. Risikofaktoren für die Entstehung von Nebenwirkungen

\begin{tabular}{lll}
\hline & Mittelwert & SD \\
\hline Patientenseitige Faktoren & & \\
Schwere der Störung & 2,91 & 0,76 \\
Dauer der Störung & 2,77 & 0,73 \\
Persönlichkeit des Patienten & 3,09 & 0,66 \\
Kritische Lebensereignisse & 2,70 & 0,79 \\
\hline Therapeutenseitige Faktoren & & \\
Diagnostik/Indikation & 3,32 & 0,67 \\
Störungsbild & 2,76 & 0,70 \\
Fallkonzeption & 3,33 & 0,62 \\
Therapiemethoden & 3,21 & 0,63 \\
Technische Fehler & 2,92 & 0,67 \\
Theoretische Qualifikation & 3,24 & 0,64 \\
Praktische Erfahrung & 2,82 & 0,81 \\
Persönliche Konflikte des Therapeuten & 3,17 & 0,72 \\
Persönlichkeit des Therapeuten & 2,93 & 0,68 \\
Unzureichende Supervision & 3,05 & 0,62 \\
Therapeut-Patient-Beziehung & 3,44 & 0,61 \\
\hline Sonstiges & & \\
Externe Faktoren & 2,69 & 0,76 \\
Gesetzliche Rahmenbedingung & 2,37 & 0,78 \\
\hline
\end{tabular}

Skalenwerte 1-4 (von "auf keinen Fall” bis “auf jeden Fall”).

\section{Ergebnisse}

Es wurden 100 Psychotherapeuten in Ausbildung interviewt. 78\% waren weiblich, das Durchschnittsalter betrug 32,2 ( $\mathrm{SD}=4,7)$ Jahre. 70\% befanden sich im 3. bzw. 4. Jahr der Ausbildung. Sie behandelten aktuell im Schnitt 7,9 (SD = 3,7) ambulante Fälle.

Von den 100 Teilnehmern der Studie erklärte eine Therapeutin zu Beginn des Interviews, dass sie davon überzeugt sei, dass Psychotherapie keine Nebenwirkungen habe. Diese Teilnehmerin nannte demzufolge auch keine Beispiele und beantwortete in der Folge keine Fragen zu den Auftretenshäufigkeiten von Nebenwirkungen.

Von den Therapeuten wurden in freier Form spontan 623 Beispiele für Nebenwirkungen genannt. Abbildung 1 zeigt den Prozentsatz an Therapeuten, von denen einzelne Nebenwirkungskategorien der UE-ATR Checkliste genannt wurden. Am häufigsten (70\% der Therapeuten) wurde die Kategorie "Verschlechterung bestehender Symptome" angesprochen, wie z.B. "Dem Patienten geht es erst mal schlechter, weil er sich mit den Problemen befasst" oder "Suizidalität". Von 59\% der Therapeuten wurden Nebenwirkungen der Kategorie "Änderung des sozialen Netzes" genannt. Beispiele dafür sind "Konflikte in Beziehungen, weil der Patient sich anders verhält, als es die Umwelt gewohnt ist" oder "Überabgrenzen". Von $54 \%$ wurde die Kategorie "Belastung durch die Therapie" erwähnt, beispielsweise "Erkenntnisse, die einem weh tun" oder "Selbstwertminderung". Beispiele der Kategorie "familiäre Veränderung" wurden von $46 \%$ genannt. $44 \%$ der Therapeuten nannten Beispiele der Kategorie "Entstehung neuer Symptome," wie z.B. "Schlafstörungen, grübeln, Schlaflosigkeit, weil ihm die Inhalte durch den Kopf gehen". 43\% sprachen die "Abhängigkeit vom Therapeuten" an, wie z.B. "Probleme bei der Ablösung" oder "Patient verliebt sich in Therapeuten". Nur ein Therapeut von 100 nannte ein Beispiel aus der Kategorie “Arbeitsunfähigkeit" durch z.B. "Berentung”. 38\% der Therapeuten nannten auch Beispiele (insgesamt 48), die nicht in eine der bestehenden UE-ATR Checklisten Kategorien einzuordnen waren, wie z.B. "überreflektieren". Die häufigsten nicht einzuordnenden Nennungen bezogen sich auf ungünstige Auswirkungen auf den Therapeuten selbst (6\%) oder Vorwürfe unethischen Therapeutenverhaltens (4\%).

Auf die Frage nach dem Anteil von Patienten, bei denen Nebenwirkungen zu erwarten sind, nannten die Therapeuten eine Rate von 55,4\% bei Psychotherapie allgemein, von $50,5 \%$ bei verhaltenstherapeutisch und von $44,9 \%$ der von ihnen selbst behandelten Fällen. Dies ist ein statistisch signifikanter Unterschied zwischen den eigenen Behandlungsfällen und Verhaltenstherapie an sich $(T(98)=3,41 ; p<0,001)$ sowie Psychotherapie allgemein $(T(98)=6,59 ; p<0,001)$.

Von den Therapeuten gaben $51,5 \%$ an, dass Verhaltenstherapie weniger Nebenwirkungen, 34,3\%, dass sie gleich viel, und 13,1\%, dass sie mehr Nebenwirkungen produziere als eine Psychoanalyse/tiefenpsychologisch fundierte Psychotherapie. Im Vergleich zur Gesprächspsychotherapie meinten nur 16,2\% der Therapeuten, dass Verhaltenstherapie weniger Nebenwirkungen habe, $49,5 \%$ gingen von gleich viel und $34,3 \%$ von mehr Nebenwirkungen aus.

Tabelle 1 zeigt, welche Faktoren die Psychotherapeuten in Ausbildung besonders für die Entstehung von Nebenwirkungen verantwortlich machen. Die Skala wurde zur statistischen Auswertung wie folgt kodiert: "auf keinen Fall" = 1, "eher nicht" $=2$, "eher ja" $=3$ und "auf jeden Fall” $=4$. Am riskantesten wurde die Patient-Therapeut-Beziehung angesehen $(\mathrm{M}=3,44, \mathrm{SD}=0,61)$, gefolgt von der Fallkonzeption $(\mathrm{M}=3,33, \mathrm{SD}=0,62)$, der Diagnostik $(M=3,32, S D=0,67)$, der fachlichen Qualifikation $(\mathrm{M}=3,24, \mathrm{SD}=0,64)$, der Therapiemethode $(\mathrm{M}=$ $3,21, \mathrm{SD}=0,63)$ und eigenen Konflikten des Therapeuten $(\mathrm{M}=3,17, \mathrm{SD}=0,72)$. Am wenigsten Bedeutung wurde externen Faktoren $(\mathrm{M}=2,69, \mathrm{SD}=0,75)$ und gesetzlichen Rahmenbedingungen $(\mathrm{M}=2,37, \mathrm{SD}=0,78)$ zugemessen.

Um herauszufinden, ob die Therapeuten eher internale oder externale Attribuierungen für das Auftreten von Nebenwirkungen wählen, wurden Mittelwerte der Items mit Therapeutenbezug (Diagnostik, Fallkonzepti- 
on, Methode, unzureichende Qualifikation, unzureichende Erfahrung, technische Fehler, unzureichende Selbsterfahrung, eigene Persönlichkeit, unzureichende Supervision) und mit Patientenbezug (Störungsbild, Schwere der Störung, Dauer der Störung, Persönlichkeit des Patienten, kritische Lebensereignisse) gebildet. Ein $T$ Test ergab einen signifikanten Unterschied $(T(98)=4,93$; $p<0,001)$ zwischen der mittleren Ursachenzuschreibung bezüglich Patientenvariablen $(\mathrm{M}=2,83, \mathrm{SD}=0,50)$ und Therapeutenvariablen $(M=3,10, S D=0,41)$. Die Verhaltenstherapeuten in Ausbildung sprechen den Therapeutenvariablen danach mehr Bedeutung bei der Verursachung von Nebenwirkungen zu als den Patientenvariablen.

\section{Diskussion}

Ein erstes Ergebnis der Untersuchung ist das Spektrum der angesprochenen Nebenwirkungen. Bei der Betrachtung von Psychotherapienebenwirkungen ist es von großer Bedeutung, nicht nur solche zu beachten, die sich auf das persönliche Wohlbefinden auswirken, sondern auch solche, die sich auf die soziale Integration des Patienten beziehen [Conrad und Auckenthaler, 2010]. Die befragten Verhaltenstherapeuten nennen mehrheitlich ein breites Spektrum an Nebenwirkungen. Etwa die Hälfte der Interviewpartner verfügt damit über ein recht gutes Grundverständnis von dem, was an Nebenwirkungen auftreten kann.

Am häufigsten erwartet werden Symptomverschlechterungen. In einer patientenbezogenen Untersuchung zeigte sich am häufigsten die emotionale Belastung durch die Therapie selbst, was aber immerhin auch von $54 \%$ der Therapeuten als Beispiel angeführt wurde [SchermulyHaupt et al., 2018]. Es gibt also keinen Hinweis auf relevante inhaltliche Verzerrungen in den Erwartungen angehender Therapeuten.

Die erwartete Häufigkeit an Fällen mit Nebenwirkungen liegt mit etwa 50\% durchaus in einem Bereich, der realistisch erscheint, und in patientenbezogenen Erhebungen gefundenen Raten [Ladwig et al., 2014; Moritz et al., 2015; Rheker et al., 2017; Cuijpers et al., 2018; Schermuly-Haupt et al., 2018]. Die Therapeuten, die sich in einer strukturierten verhaltenstherapeutischen Ausbildung befinden, scheinen sich im Durchschnitt, wenn man von Einzelfällen absieht, durchaus der Nebenwirkungsproblematik bewusst zu sein. Dies ist bemerkenswert, da es in keinem der Institute bis dahin spezielle Kurse zu diesem Thema gab, und spricht für die Güte der Grundausbildung und Supervision an sich.

Interessant ist der Hinweis aus den Daten auf den in der Literatur vielfach beschriebenen Bias bezüglich der Einschätzung der Risiken der eigenen Therapien. Es ist offenbar wie beim Autofahren. Unfälle machen immer die anderen, da man über den eigenen Fahrstil ja Kontrolle hat. Die Therapeuten zeigen einen positiven Bias gegenüber der eigenen Therapie. Eine Erklärung für derartige Befunde bietet die Sozialpsychologie, die gezeigt hat, dass Menschen grundsätzlich dazu neigen, sich selbst und die eigenen Fähigkeiten positiv wahrzunehmen. Inkonsistenzen führen zu kognitiven Dissonanzen, die Spannungszustände auslösen, die das Wohlbefinden negativ beeinflussen können. Deswegen sind Menschen motiviert, diese Dissonanzen aufzulösen, was aber auch die Fähigkeit einschränkt, die eigenen Kompetenzen richtig einzuschätzen [Festinger, 1962; Mezulis et al., 2004]. Es kann des Weiteren auch vermutet werden, dass Nebenwirkungen von Psychotherapie grundsätzlich auch eine Bedrohung des Selbstwerts von Therapeuten darstellen und daher abgewehrt werden.

Nicht ganz so eindeutig ist die grundsätzliche Einschätzung des eigenen Psychotherapieverfahrens Verhaltenstherapie im Vergleich zu anderen Verfahren. Die psychodynamischen Verfahren werden eindeutig als riskanter angesehen, während die klientenzentrierte Psychotherapie eher als risikoärmer beurteilt wird. Dazu gibt es unseres Wissens nur wenige Daten, sodass sich die Frage stellt, welche Wahrnehmungs- und Erfahrungsschemata zu diesen Einschätzungen führen. In einer Fragebogenuntersuchung an Patienten von Leitner et al. [2013] scheinen die humanistischen und systemischen Psychotherapien tatsächlich seltener zu unerwünschten Ereignissen in der Therapie zu führen. Dies scheint ein interessantes Thema für zukünftige verfahrensvergleichende Untersuchungen zu sein.

Die Befragung zu den Ursachen von Nebenwirkungen zeigt, dass die Therapeuten sich des Zusammenhangs zwischen Nebenwirkung und eigenem Verhalten bewusst sind. Als bedeutsam sehen sie die Therapeut-Patient-Beziehung und dabei vor allem das eigene Verhalten. Dies ist auch bemerkenswert, da die Befragten in der Mehrheit offenbar nicht einem möglichen Risiko verfallen, alle Negativentwicklungen in der Therapie oder im Zustand des Patienten diesem zuzuschreiben [Schermuly et al., 2014]. Ähnlich selbstkritisch hatten sich auch die Therapeuten in der Untersuchung zum Schadensverständnis von Gesprächspsychotherapeuten von Frohburg [2002] geäußert.

Castonguay und Kollegen [2010] haben einige Vorschläge erarbeitet, wie man Therapeuten in Ausbildung helfen kann, negativen Effekten und Nebenwirkungen vorzubeugen. Voraussetzung ist, sich als Therapeut nicht vor negativen Effekten zu fürchten, sondern diese als unvermeidliche Begleiteffekte einer guten und erfolgreichen Therapie zu verstehen, auf die auch therapeutisch einzugehen ist. Es ist auch wichtig, auf Patientenseite realistische Erwartungen bezüglich der Chancen und Risiken 
von Psychotherapie aufzubauen [Seligman et al., 2009; Rief und Glombiewski, 2016]. Dies ist nicht nur das Recht der Patienten, sondern kann auch helfen, Kooperationsproblemen vorzubeugen und eine gute Arbeitsbeziehung aufzubauen und $\mathrm{zu}$ erhalten. Psychotherapie ist keine Kunst, sondern eine Wissenschaft, zu der eine offene und transparente Fehlerkultur gehört [Caspar und Kächele, 2008], die das Fach noch professioneller macht und letztendlich auch dadurch noch mehr Patienten helfen kann.

Die vorliegende Untersuchung hat einige Begrenzungen, die bei der Interpretation der Daten zu bedenken sind. Sie bezieht sich nur auf Verhaltenstherapeuten und ist nicht ohne Weiteres auf sonstige Therapieverfahren übertragbar. Den Therapeuten war das Thema der Befragung erkennbar, was das Antwortverhalten beeinflusst haben kann. Die Therapeuten wurden nicht randomisiert ausgewählt oder systematisch rekrutiert, sondern haben sich nach einer allgemeinen Information freiwillig zu einer Mitarbeit bereit erklärt. Es könnte also sein, dass ein Rekrutierungsbias derart vorliegt, dass nur die besonders gewissenhaften Ausbildungskandidaten teilgenommen haben oder möglicherweise genau die Therapeuten Interesse an der Untersuchung zeigten, die bereits in ihrer eigenen Arbeit oder der Ausbildung Erfahrungen mit dem Thema Nebenwirkungen gesammelt hatten, was die Ergebnisse in Richtung einer hohen Sensibilität verzerrt haben könnte. Ebenso stammen die Teilnehmer aus drei sehr etablierten Instituten und sind insofern nur prototypisch repräsentativ für gleichartige Institute.

Trotz dieser Einschränkungen können aus den Daten mehrere Schlussfolgerungen für die Therapeutenausbildung gezogen werden:

(1) Die Häufigkeitsangaben zu erwarteten Nebenwirkungen zeigen, dass die Therapieausbildung in Theorie und vor allem auch in der supervidierten Fallbehandlung durchaus schon eine Offenheit für das Thema schaffen kann. Dies ist ein positiver Ausgangspunkt für eine weiterführende systematische Abhandlung in der Ausbildung, in der das Handwerkszeug und eine Haltung vermittelt wird, wie mit Nebenwirkungen umgegangen werden kann und auch wie Patienten über diese aufgeklärt werden können.

(2) Der festgestellte selbstbezogene positive Wahrnehmungsbias muss didaktisch so angegangen werden, dass das Thema selbstwertstabilisierend und nicht verunsichernd abgehandelt wird. Dies kann im Rahmen von Gruppensupervisionen erfolgen. Wenn es einem Therapeuten schwerfällt, in den eigenen Therapien nachteilige Begleiteffekte auszumachen, so kann er bei der Supervision der Fälle der Kollegen dafür sensibilisiert werden. Es konnte auch gezeigt werden, dass ein zeitnahes und konsequentes Feedback über den Fortschritt von Patienten im Therapieverlauf die Ergebnisse der Behandlung verbessert und gleichzeitig Patienten mit nachteiligen Ent- wicklungen leichter identifiziert werden können [Macdonald und Mellor-Clark, 2015].

(3) Bei der Ausbildung und der Erfassung im konkreten Fall kann eine systematische Gruppierung und Analyse hilfreich sein. Dies kann beispielsweise in Anlehnung an die UE-ATR Checkliste [Linden und Haupt, 2012; Linden, 2013] geschehen, die dazu zwingt, die Bereiche und die psychotherapeutischen Prozesse zu inspizieren, in denen oder durch die Nebenwirkungen auftreten können.

Zusammenfassend ergeben die erhobenen Befunde ein positives Gesamtbild. Die Ausbildungskandidaten haben zu dem Thema der Psychotherapienebenwirkungen bereits gute Kenntnisse und Einstellungen, auf denen eine systematische Lehre aufbauen kann.

\section{Dank}

Mein Dank gilt den Ausbildungsinstituten IVB, ZPHU und IVT, die sich an der Untersuchung beteiligt haben, und jedem einzelnen Therapeuten, der interessiert und aufgeschlossen an der Befragung teilgenommen hat. Ohne die konstruktive Haltung der Institutsleiter und ihrer Ausbildungskandidaten wäre die Untersuchung nicht möglich gewesen.

\section{Ethische Aspekte}

Es handelt sich um eine Befragung von Psychotherapeuten zu ihrem Berufsfeld ohne persönliche Einbeziehung von Patienten oder Einflussnahme auf laufende Behandlungen. Die Befragungsteilnehmer wurden über die Ziele der Befragung informiert und konnten jederzeit eine Teilnahme ohne irgendwelche Konsequenzen ablehnen. Die Studie wurde von den Institutsleitungen geprüft und bewilligt und es wurden alle ethischen Standards der Deklaration von Helsinki eingehalten.

\section{Disclosure Statement}

Die Erstautorin ist approbierte Psychologische Psychotherapeutin und hat die Datenerhebung und wesentliche Teile der Auswertungen als Teil ihrer Dissertationsarbeit durchgeführt. Der Zweitautor ist u.a. Dozent und Supervisor am Institut für Verhaltenstherapie Berlin und hat wesentlich an der Entwicklung des Studienkonzepts mitgewirkt. Beide Autoren haben bei der Erstellung des Manuskripts zusammengearbeitet. Seitens der Leitungen der Institute erfolgte keine Einflussnahme. Die Autoren erklären, dass keine Interessenkonflikte bestehen.

\section{Finanzielle Unterstützung}

Es existieren keine finanziellen Zuwendungen durch Firmen oder andere Personen/Institutionen, die in irgendeiner Weise diesen Artikel oder dessen Veröffentlichung beeinflussen können. 


\section{Literatur}

Barlow DH. Negative effects from psychological treatments: a perspective. Am Psychol. 2010 Jan;65(1):13-20.

Berk M, Parker G. The elephant on the couch: sideeffects of psychotherapy. Aust N Z J Psychiatry. 2009 Sep;43(9):787-94.

Boisvert CM, Faust DF. Practising psychologist's knowledge of general psychotherapy research findings. Prof Psychol Res Pr. 2006;37:708-16.

Brakemeier EL, Breger V, Spitzer C. Nebenwirkungen von ambulanter und stationärer Psychotherapie. Verhaltensther Psychosoz Prax. 2012;44:489-510.

Bundespsychotherapeutenkammer (BPTK) (2013): Informationen zum Patientenrechtegesetz für Psychotherapeuten und Psychotherapeutinnen. http://bptk.de/fileadmin/ user_upload/Publikationen/BPtK_Infomaterial/Patientenrechtegesetz/20130923_bptkInfomaterial_Patientenrechtegesetz.pdf.

Bystedt S, Rozental A, Andersson G, Boettcher J, Carlbring P. Clinicians' perspectives on negative effects of psychological treatments. Cogn Behav Ther. 2014;43(4):319-31.

Caspar F, Kächele H. Fehlentwicklungen in der Psychotherapie. In: Herpertz SC, Caspar F, Mundt C, editors. Störungsorientierte Psychotherapie. München: Elsevier; 2008. pp. 729-43.

Castonguay LG, Boswell JF, Constantino MJ, Goldfried MR, Hill CE. Training implications of harmful effects of psychological treatments. Am Psychol. 2010 Jan;65(1):34-49.

Conrad A, Auckenthaler A. Therapiemisserfolge in ambulanter Einzeltherapie: Die Sicht der Klienten. Psychother Soz wiss. 2010;10:7-41.

Cuijpers P, Reijnders M, Karyotaki E, de Wit L, Ebert DD. Negative effects of psychotherapies for adult depression: A meta-analysis of deterioration rates. J Affect Disord. 2018 Oct;239: 138-45.

Dishion TJ, McCord J, Poulin F. When interventions harm. Peer groups and problem behavior. Am Psychol. 1999 Sep;54(9):755-64.

Dobson K, Beshai S. The theory-practice gap in cognitive behavioral therapy: reflections and a modest proposal to bridge the gap. Behav Ther. 2013 Dec;44(4):559-67.

Dunning D, Heath C, Suls JM. Flawed self-assessment: implications for health, education, and the workplace. Psychol Sci Public Interest. 2004 Dec;5(3):69-106.

Festinger L. A theory of cognitive dissonance. Stanford: Stanford Univ. Press; 1962.

Frohburg I. Untersuchung zum Schadensverständnis von GesprächspsychotherapeutInnen. In: Märtens $\mathrm{M}$, Petzold $\mathrm{H}$, editors. Therapieschäden. Mainz: Matthias Grünewald-Verlag; 2002. pp. 72-108.

Hannan C, Lambert MJ, Harmon C, Nielsen SL, Smart DW, Shimokawa K, et al. A lab test and algorithms for identifying clients at risk for treatment failure. J Clin Psychol. 2005 Feb 61(2):155-63.

Hasenauer M, Tschuschke V. Effektivität und Erfolgsprädiktoren in der kognitiven Verhaltenstherapie unter Praxisbedingungen. Verhaltenstherapie. 2017;27(2):86-95.

Hatfield D, McCullough L, Frantz SH, Krieger K. Do we know when our clients get worse? an investigation of therapists' ability to detect negative client change. Clin Psychol Psychother. 2010 Jan-Feb;17(1):25-32.

Hermanutz M. Falsche Erinnerungen: Ein Problem auch in der Psychotherapie? Verhaltenstherapie. 2017;27(4):272-81.

Jacobi F, Uhmann S, Hoyer J. Wie häufig ist therapeutischer Misserfolg in der ambulanten Psychotherapie? Z Klin Psychol Psychother. 2011; 40(4):246-56.

Ladwig I, Rief W, Nestoriuc Y. Welche Risiken und Nebenwirkungen hat Psychotherapie? Entwicklung des Inventars zur Erfassung Negativer Effekte von Psychotherapie (INEP). Verhaltenstherapie. 2014;24(4):252-63.

Lambert MJ, Ogles BM. The efficacy and effectiveness of psychotherapy. Lambert MJ (Hg): Bergin and Garfield's Handbook of Psychotherapy and Behavior Change. 5th ed. New York: Wiley; 2004. pp. 139-93.

Lambert MJ. What have we learned about treatment failure in empirically supported treatments? Some suggestions for practice. Cognit Behav Pract. 2011;18(3):413-20.

Lambert MJ. Predicting negative treatment outcome: Methods and estimates of accuracy. In: Lambert MJ, editor. Prevention of treatment failure: The use of measuring, monitoring, and feedback in clinical practice. Washington: American Psychological Association; 2010. pp. 83-105.

Leitner A, Märtens M, Koschier A, Gerlich K, Liegl $\mathrm{G}$, Hinterwallner $\mathrm{H}$, et al. Patients perceptions of risky developments during psychotherapy. J Contemp Psychother. 2013;43(2):95-105.

Lilienfeld SO. Psychological treatments that cause harm. Perspect Psychol Sci. 2007 Mar;2(1):5370 .

Linden M, Haupt ML. Erfassung von Nebenwirkungen in der Psychotherapie. In: Linden M, Strauß B, editors. Risiken und Nebenwirkungen von Psychotherapie: Erfassung, Bewältigung, Risikovermeidung. Berlin: MWV Medizinisch Wissenschaftliche Verlagsgesellschaft; 2012. pp. 59-74.

Linden M, Strauss B, Scholten S, et al. Definition und Entscheidungsschritte in der Bestimmung und Erfassung von Nebenwirkungen von Psychotherapie. Psychother Psych Med. 2018; 68(09/10): 377-82. DOI: https://doi. org/10.1055/a-0619-5949.

Linden M, Strauss B. Risiken und Nebenwirkungen von Psychotherapie. Erfassung, Bewältigung, Risikovermeidung. 2. aktualisierte Auflage. Berlin: Medizinisch Wissenschaftliche Verlagsgesellschaft; 2018

Linden M, Walter M, Fritz K, Muschalla B. Unerwünschte Therapiewirkungen bei verhaltenstherapeutischer Gruppentherapie: Häufigkeit und Spektrum. Nervenarzt. 2015 Nov;86(11): 1371-82.

Linden M. How to define, find and classify side effects in psychotherapy: from unwanted events to adverse treatment reactions. Clin Psychol Psychother. 2013 Jul-Aug;20(4):286-96.

Macdonald J, Mellor-Clark J. Correcting psychotherapists' blindsidedness: formal feedback as a means of overcoming the natural limitations of therapists. Clin Psychol Psychother. 2015 May-Jun;22(3):249-57.

Mayou RA, Ehlers A, Hobbs M. Psychological debriefing for road traffic accident victims.
Three-year follow-up of a randomised controlled trial. Br J Psychiatry. 2000 Jun;176(6): 589-93

Mezulis AH, Abramson LY, Hyde JS, Hankin BL. Is there a universal positivity bias in attributions? A meta-analytic review of individual, developmental, and cultural differences in the self-serving attributional bias. Psychol Bull. 2004 Sep;130(5):711-47.

Moos RH. Iatrogenic effects of psychosocial interventions for substance use disorders: prevalence, predictors, prevention. Addiction. 2005 May;100(5):595-604.

Moritz S, Fieker M, Hottenrott B, Seeralan T, Cludius B, Kolbeck K, et al. No pain, no gain? Adverse effects of psychotherapy in obsessivecompulsive disorder and its relationship to treatment gains. J Obsessive Compuls Relat Disord. 2015;5:61-6.

Nelson NE, Hiller W. Therapeutischer Misserfolg in der ambulanten Psychotherapie. Z Klin Psychol Psychother. 2013;42(4):217-29.

Pence SL Jr, Sulkowski ML, Jordan C, Storch EA. When exposures go wrong: troubleshooting guidelines for managing difficult scenarios that arise in exposure-based treatment for obsessive-compulsive disorder. Am J Psychother. 2010;64(1):39-53.

Rheker J, Beisel S, Kräling S, Rief W. Rate and predictors of negative effects of psychotherapy in psychiatric and psychosomatic inpatients. Psychiatry Res. 2017 Aug;254:143-50.

Rief W, Glombiewski JA. Erwartungsfokussierte Psychotherapeutische Interventionen (EFPI). Verhaltenstherapie. 2016;26(1):47-54.

Roback HB. Adverse outcomes in group psychotherapy: risk factors, prevention, and research directions. J Psychother Pract Res. 2000;9(3): 113-22.

Schermuly CC, Schermuly-Haupt ML, Schölmerich F, Rauterberg H. Zu Risiken und Nebenwirkungen lesen Sie. Negative Effekte von Coaching. Z Arb Organpsychol. 2014;58(1): 17-33.

Schermuly-Haupt M, Linden M, Rush A. Unwanted events and side effects in cognitive behavior therapy. Cognit Ther Res. 2018;42(3):219-29.

Seligman LD, Wuyek LA, Geers AL, et al. The effects of inaccurate expectations on experiences with psychotherapy. Cognit Ther Res. 2009; 33(2):139-49.

Strauß B, Eckert J. Schäden und negative Folgen von Gruppenpsychotherapien. Gruppenpsychother Gruppendyn. 2001;37:45-67.

Walfish S, McAlister B, O’Donnell P, Lambert MJ. An investigation of self-assessment bias in mental health providers. Psychol Rep. 2012 Apr;110(2):639-44.

Warren JS, Nelson PL, Mondragon SA, Baldwin SA, Burlingame GM. Youth psychotherapy change trajectories and outcomes in usual care: community mental health versus managed care settings. J Consult Clin Psychol. 2010 Apr; 78(2):144-55.

Wirtz MA, Caspar F. Beurteilerübereinstimmung und Beurteilerreliabilität: Methoden zur Bestimmung und Verbesserung der Zuverlässigkeit von Einschätzungen mittels Kategoriensystemen und Ratingskalen. Göttingen: Hogrefe; 2002. 